\title{
Determining Best Patch Management Software using Intuitionistic Fuzzy Sets with TOPSIS
}

\author{
Yogita Kansal $^{\mathrm{a}, *}$, P. K. Kapur ${ }^{\mathrm{b}}$, and Nitin Sachdeva ${ }^{\mathrm{c}}$ \\ ${ }^{a}$ Amity Institute of Information Technology, Amity University Uttar Pradesh, Noida, 201313, India \\ ${ }^{b}$ Amity Centre for Interdisciplinary Research, Amity University Uttar Pradesh, Noida, 201313, India \\ 'Institute of Management Technology (IMT), Ghaziabad, 201001, India
}

\begin{abstract}
Today's IT infrastructure demands for an automated yet stringently controlled solution to manage patches for vulnerable software applications. The use of patch management tools is the best practice that tests all the available patches before installation to ensure that the released patch will not break the existing software. However, the availability of several patch management software poses a challenge for the system administrator to decide which software facilitates the operational competence and effectiveness of the computer system in terms of revenue and system security. Therefore, selecting the appropriate patch management software that automatically patches all the Microsoft and non-Microsoft products simultaneously is an important and complex concern, leading to the multi-criteria decision approach. Here, we implement a hybrid approach that combines the intuitionistic fuzzy set and entropy weight-based multi-criteria decision making model with TOPSIS to select the best defense against vulnerabilities (or patch management software) in the group decision making environment. As most real world decision problems involve a group of decision makers that may have multiple opinions for individual criteria, the intuitionistic fuzzy weighted averaging operator is explicitly considered here and generates optimal weights for the attributes. A numerical example is provided to illustrate the application of the intuitionistic fuzzy TOPSIS method that helps identify the best patch management tool based on selected criteria.
\end{abstract}

Keywords: vulnerability; patch management; multi-criteria decision method; intuitionistic fuzzy; entropy; TOPSIS

(Submitted on November 18, 2017; Revised on March 7, 2018; Accepted on April 10, 2018)

(C) 2019 Totem Publisher, Inc. All rights reserved.

\section{Introduction}

Patch management (PM) has recently received considerable attention in both small- and large-scale enterprises. The PM is performed mainly by software companies as part of their internal efforts to solve problems with different software versions, to analyze current software programs, and to detect any possible deficiencies in security features or other upgrades. The way patches are distributed and deployed has experienced extreme changes over time. Conventionally, patches were provided on external media which could then be added to previously installed programs. Today, however, with the availability of Internet and cloud-hosting systems, everything has become discrete. Nowadays, there is no need for external media; rather, patches can be applied to software programs directly over the global IP network. This is the era of automated patch management software.

One of the major activities for PM success is technology or software that provides assistance in the completion of patch management tasks [1]. Technology notifies the availability of patches from a third-party vendor, deploys the patches onto vulnerable systems, maintains operational efficiency and effectiveness, overcomes security vulnerabilities, and maintains the stability of the production environment [2]. The most important aspect of technology is the selection of appropriate patch management software that balances the security and downtime risk of a security breach.

One of the most well-known studies [3] identified five important evaluation criteria that businesses should keep in mind when selecting the right patch management software. There are eight criteria or factors for selecting the secure patch

\footnotetext{
* Corresponding author.

E-mail address: ykansal35@gmail.com
} 
management software [4].

In this paper, our major criteria on which scrutiny is performed are as follows: vulnerability prioritization, vulnerability analysis, and vendor participation. Recent studies suggest that almost $60 \%$ of organizations are not fully capable of identifying vulnerabilities that need updates. Thus, a patch management tool must be efficient in prioritizing vulnerabilities and patches according to the host environment. The second criterion addresses the need for risk assessment that determines the consequences of patch deployment (or change management) and develops hot fixes and walkthroughs automatically. The last criterion, i.e., vendor participation, encompasses all the third-party updates and patches relevant to the individuals' organization. All three criteria address the need for both IT security and IT operations.

The top five patch management software are taken here as alternatives, out of which the best tool that fulfils all three criteria is selected. The software are Solar Winds, Shavlik, LanDesk PM, Manage Engine Desktop Central, and GFI LanGuard. SolarWinds centralizes the entire patch process, from download to publish, and then to patch. It is integrated with Windows server update services and Microsoft update agent that automates patching on the basis of custom schedules [5]. Shavlik offers agentless patching such that patches are deployed to users' physical and virtual assets [6]. It performs well in large environments and maximizes users' effort by adding third-party patching to the system center configuration manager. Manage Engine integrates desktop and mobile devices to support Windows, Mac, and Linux as well as iOS and Android [7]. Built-in templates make it easy to manage patch installation for package creation. GFI LanGuard has the best user interface that supports many operating systems such as Mac OS X, Microsoft, Linux, and more than 60 third-party applications. It discovers risks and threats early to provide automate remediation for dynamic environments [8].

We propose a hybrid intuitionistic fuzzy set (IFS) entropy-based multi-criteria group decision making with the "Technique for Order of Preference by Similarity to Ideal Solution (TOPSIS)" method developed in [9] to select appropriate patch management software. Since it is a challenging issue to express the views of experts for criteria and the alternatives using crisp data, IFS is an effective way to implement multiple decision-making problems in an uncertain environment. We have considered the "intuitionistic fuzzy weighted averaging (IFWA) operator" to aggregate the opinions of all individuals such that the importance of each criteria and the impact of alternatives on criteria are ranked. On the other hand, TOPSIS provides both positive and negative ideal solutions for the multi-attribute decision making problem. Hence, it is assumed that the probability of success for patch management tool selection is high when TOPSIS is combined with IFS.

The paper is organized as follows: a detailed description of the proposed hybrid intuitionistic fuzzy with entropy and TOPSIS is presented in section 2. Section 3 demonstrates a case study of the proposed methodology, followed by the conclusion in Section 4.

\section{Proposed Methodology}

\subsection{Preliminaries}

\subsubsection{Intuitionistic Fuzzy Set}

Intuitionistic fuzzy set is an extension of the classical fuzzy set theory that was first introduced [10] to tackle fuzziness seen in the decision-making process. In a finite set $P$, an intuitionistic fuzzy set $M$ is defined as:

$$
M=\left\{\left(p, \mu_{M}(p), u_{M}(p)\right) \mid p \in P\right\}
$$

Where $\mu_{M}(p)$ is the membership function and $u_{M}(p): P \rightarrow[0,1]$ is the non-membership function, with the condition that

$$
0 \leq \mu_{M}(p)+u_{M}(p) \leq 1
$$

For every IFS $M$ in $P$, the third parameter $\pi_{M}(p)$ is known as the intuitionistic fuzzy index or hesitation degree of whether p belongs to $M$ or not.

$$
\pi_{M}(p)=1-\mu_{M}(p)-u_{M}(p)
$$


It is obviously seen that for every $p \in P, 0 \leq \pi_{M}(p) \leq 1$.

If $\pi_{M}(p)$ is small, information regarding $p$ is more definite; meanwhile, this is not true when $\pi_{M}(p)$ becomes large. When $\mu_{M}(p)=1-u_{M}(p) \forall p$, the regular fuzzy set theory is improved [11]. Let $M$ and $N$ be the IFSs of the set $P$, and then multiplication operator is defined as follows [10]:

$$
M \otimes N=\left\{\mu_{M}(p) \cdot \mu_{N}(p), u_{M}(p)+u_{N}(p) \cdot \pi_{M}(p) \cdot \pi_{N}(p) \mid p \in P\right\}
$$

\subsubsection{Entropy of IFS}

The entropy function [12] measures the discrete distribution uncertainty on the basis of classical statistical mechanics known for Boltzmann entropy. [13] proposed a Shannon's function based the non-probabilistic entropy formula of a fuzzy set on a finite universal set as $P=\left\{p_{1}, p_{2}, p_{3}, \cdots, p_{k}\right\}$.

$$
E_{L T}(M)=-i \sum_{x=1}^{k}\left[\mu_{M}\left(p_{x}\right) \ln \mu_{M}\left(p_{x}\right)+\left(1-\mu_{M}\left(p_{x}\right)\right) \ln \left(1-\mu_{M}\left(p_{x}\right)\right)\right], i>0
$$

The work conducted in [13] was later extended in [14]. The authors provided multiple descriptions for entropy measure on IFSs(P). [15] measured the intuitionistic fuzzy entropy through the following equation, which satisfies the four axiomatic constraints:

$$
E_{L T}^{I F S}(M)=-\frac{1}{k \ln 2} \sum_{x=1}^{k}\left[\mu_{M}\left(p_{x}\right) \ln \mu_{M}\left(p_{x}\right)+u_{M}\left(p_{x}\right) \ln u_{M}\left(p_{x}\right)-\left(1-\pi_{M}\left(p_{x}\right)\right) \ln \left(1-\pi_{M}\left(p_{x}\right)\right)-\pi_{M}\left(p_{x}\right) \ln 2\right]
$$

It is noted that $E_{L T}^{I F S}(M)$ is composed of the hesitancy degree and the fuzziness degree of the IFS $M$.

\subsection{Proposed Intuitionistic Fuzzy and Entropy-based Decision Model with TOPSIS}

Let $M=\left\{M_{1}, M_{2}, \cdots, M_{a}\right\}$ denote the set of alternatives and $P=\left\{P_{1}, P_{2}, \cdots, P_{k}\right\}$ denote the set of criteria. Here, we explain the step by step procedure for the intuitionistic fuzzy and entropy-based decision model with the TOPSIS method as shown in Figure 1.

\section{Stage 1. Determine the weights of decision makers}

If we have ' $X$ ' decision makers, then their importance is determined through linguistic terms expressed in intuitionistic fuzzy numbers.

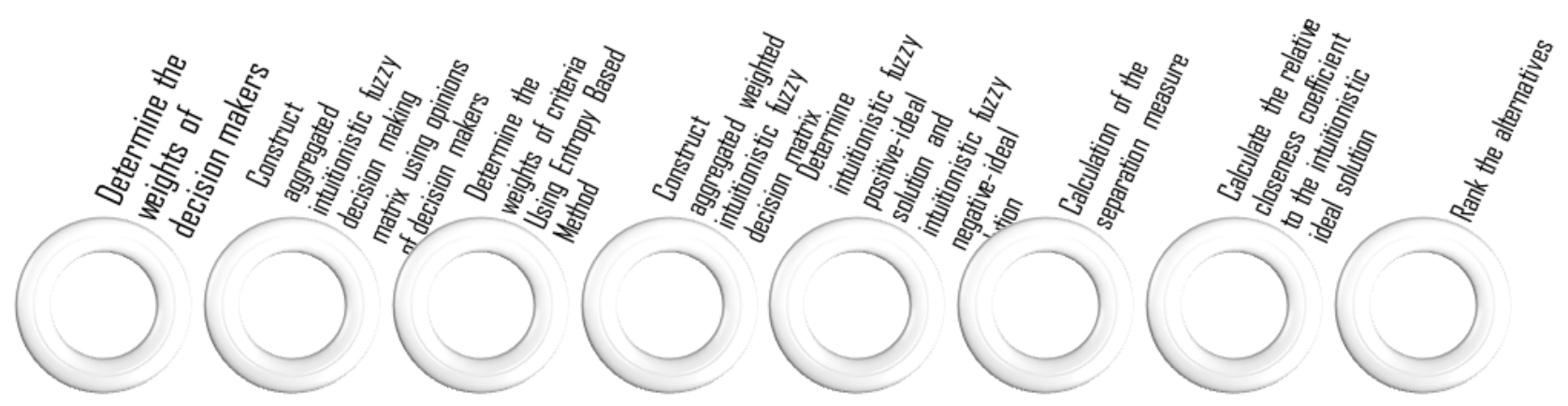

Figure 1. Steps for implementation of proposed model

If $D_{i}=\left(\mu_{i}, u_{i}, \pi_{i}\right)$ is an intuitionistic fuzzy number that ranks the $i^{\text {th }}$ decision maker, then the weight of $i^{\text {th }}$ decision maker can be obtained as: 


$$
\phi_{i}=\frac{\left(\mu_{i}+\pi_{i}\left(\frac{\mu_{i}}{\mu_{i}+u_{i}}\right)\right)}{\sum_{i=1}^{l}\left(\mu_{i}+\pi_{i}\left(\frac{\mu_{i}}{\mu_{i}+u_{i}}\right)\right)}, \sum_{i=1}^{l} \phi_{i}=1
$$

\section{Stage 2. Construct an aggregated intuitionistic fuzzy decision matrix based on the opinions of decision makers}

Let $U^{(i)}=\left(u_{x y}^{(i)}\right)_{a \times k}$ be an intuitionistic fuzzy decision matrix of each decision maker. $\phi=\left\{\phi_{1}, \phi_{2}, \phi_{3}, \cdots, \phi_{l}\right\}$ is the weight of each decision maker, and $\sum_{i=1}^{l} \phi_{i}=1, \phi_{i} \in[0,1]$. In the process of group decision-making, all the individual opinions are consolidated into a group opinion such that an aggregated intuitionistic fuzzy decision matrix is created via the IFWA operator proposed in [16].

$$
U=\left(u_{x y}\right)_{a \times k}
$$

Where

$$
\begin{aligned}
u_{x y} & =I F W A_{\phi}\left(u_{x y}^{1}, u_{x y}^{2}, \cdots, u_{x y}^{l}\right) \\
& =\phi_{1} u_{x y}^{1} \oplus \phi_{2} u_{x y}^{2} \oplus \cdots \oplus \phi_{l} u_{x y}^{l} \\
& =\left[1-\prod_{i=1}^{l}\left(1-\mu_{x y}^{(i)}\right)^{\phi_{i}}, \prod_{i=1}^{l}\left(\mu_{x y}^{(i)}\right)^{\phi_{i}}, \prod_{i=1}^{l}\left(1-\mu_{x y}^{(i)}\right)^{\phi_{i}}-\prod_{i=1}^{l}\left(\mu_{x y}^{(i)}\right)^{\phi_{i}}\right]
\end{aligned}
$$

Here, $u_{x y}=\left(\mu_{M_{x}}\left(p_{y}\right), \pi_{M_{x}}\left(p_{y}\right)\right),(x=1,2, \cdots, a ; y=1,2, \cdots, k)$.

The aggregated intuitionistic fuzzy decision matrix can be defined as follows:

$$
\begin{gathered}
U=\left[\begin{array}{cccc}
\left(\mu_{M_{1}}\left(p_{1}\right), u_{M_{1}}\left(p_{1}\right), \pi_{M_{1}}\left(p_{1}\right)\right) & \left(\mu_{M_{1}}\left(p_{2}\right), u_{M_{1}}\left(p_{2}\right), \pi_{M_{1}}\left(p_{2}\right)\right) & \cdots & \left(\mu_{M_{1}}\left(p_{n}\right), u_{M_{1}}\left(p_{n}\right), \pi_{M_{1}}\left(p_{n}\right)\right) \\
\left(\mu_{M_{2}}\left(p_{1}\right), u_{M_{2}}\left(p_{1}\right), \pi_{M_{2}}\left(p_{1}\right)\right) & \left(\mu_{M_{2}}\left(p_{2}\right), u_{M_{2}}\left(p_{2}\right), \pi_{M_{2}}\left(p_{2}\right)\right) & \cdots & \left(\mu_{M_{2}}\left(p_{n}\right), u_{M_{2}}\left(p_{n}\right), \pi_{M_{2}}\left(p_{n}\right)\right) \\
\vdots & \vdots & \vdots & \vdots \\
\left(\mu_{M_{a}}\left(p_{1}\right), u_{M_{a}}\left(p_{1}\right), \pi_{M_{a}}\left(p_{1}\right)\right) & \left(\mu_{M_{a}}\left(p_{2}\right), u_{M_{a}}\left(p_{2}\right), \pi_{M_{a}}\left(p_{2}\right)\right) & \cdots & \left(\mu_{M_{a}}\left(p_{n}\right), u_{M_{a}}\left(p_{n}\right), \pi_{M_{a}}\left(p_{n}\right)\right)
\end{array}\right] \\
U=\left[\begin{array}{cccc}
u_{11} & u_{12} & \cdots & u_{1 k} \\
u_{21} & u_{22} & \cdots & u_{2 k} \\
\vdots & \vdots & \vdots & \vdots \\
u_{a 1} & u_{a 2} & \cdots & u_{a k}
\end{array}\right]
\end{gathered}
$$

\section{Stage 3. Determine the criteria weights using the entropy-based method}

Let $q_{y}^{i}=\left\{\mu_{y}^{(i)}, u_{y}^{(i)}, \pi_{y}^{(i)}\right\}$ be an intuitionistic fuzzy number assigned to criterion $P_{j}$ by the $i^{\text {th }}$ decision maker. Then, the weights of the criteria are calculated by using the well-known entropy method [17].

$$
E_{L T}^{I F S}\left(C_{y}\right)=-\frac{1}{a \ln 2} \sum_{x=1}^{a}\left[\mu_{x y}\left(C_{x}\right) \ln \mu_{x y}\left(C_{x}\right)+u_{x y}\left(C_{x}\right) \ln u_{x y}\left(C_{x}\right)-\left(1-\pi_{x y}\left(C_{x}\right)\right) \ln \left(1-\pi_{x y}\left(C_{x}\right)\right)-\pi_{x y}\left(C_{x}\right) \ln 2\right]
$$

Where $y=1,2, \cdots, k$ and $1 /(a \ln 2)$ is a constant, which assures $0 \leq E_{L T}^{I F S}\left(C_{y}\right) \leq 1$. 
Therefore, the degree of divergence $\left(d_{y}\right)$ of the average intrinsic information provided by the corresponding performance ratings on criterion $C_{y}$ can be defined as:

$$
\left(d_{y}\right)=1-E_{L T}^{I F S}\left(C_{y}\right), y=1,2, \cdots, k
$$

The value of $\left(d_{y}\right)$ represents the inherent contrast intensity of criterion $C_{y}$, and then the entropy weight of the $y^{\text {th }}$ criterion is:

$$
w_{y}=\frac{d_{y}}{\sum_{y=1}^{k} d_{y}}
$$

\section{Stage 4. Construct an aggregated weighted intuitionistic fuzzy decision matrix}

After the weights of criteria $(W)$, the aggregated weighted intuitionistic fuzzy decision matrix is constructed based on the following definition:

$$
\begin{gathered}
\bar{E}=W^{T} \otimes \bar{D}=W^{T} \otimes\left[\bar{p}_{x y}\right]_{a \times k}=\left[\overline{\bar{p}}_{x y}\right] \\
W=\left(w_{1}, w_{2}, \cdots, w_{y}, \cdots, w_{k}\right) \\
\overline{\bar{p}}_{x y}=\left\langle\overline{\bar{\mu}}_{x y}, \overline{\bar{u}}_{x y}, \overline{\bar{\pi}}_{x y}\right\rangle=\left\langle 1-\left(1-\mu_{x y}\right)^{w_{x}}, u_{x y}^{w_{x}}, 1-\left(1-\left(1-\mu_{x y}\right)^{w_{x}}-u_{x y}^{w_{x}}\right)\right\rangle, w_{y}>0
\end{gathered}
$$

Stage 5. Determine the intuitionistic fuzzy positive ideal solution (IFPIS) and intuitionistic fuzzy negative ideal solution (IFNIS)

In general, the evaluation criteria $Y_{1}$ and $Y_{2}$ are the benefit criteria and cost criteria, respectively. $A^{+}$is an intuitionistic fuzzy positive ideal solution, and $A^{-}$is an intuitionistic fuzzy negative ideal solution. The IFS theory and standard of the conventional TOPSIS method defines IFPIS and IFNIS as:

$$
\begin{aligned}
& A^{+}=\left(\overline{\bar{\mu}}_{A^{+} W}\left(C_{y}\right), \overline{\bar{u}}_{A^{+} W}\left(C_{y}\right)\right) \text { and } A^{-}=\left(\overline{\bar{\mu}}_{A^{-} W}\left(C_{y}\right), \overline{\bar{u}}_{A^{-} W}\left(C_{y}\right)\right) \\
& \overline{\bar{\mu}}_{A^{+} W}\left(C_{y}\right)=\left(\left(\max \overline{\bar{\mu}}_{A^{+} W}\left(C_{y}\right) \mid y \in Y_{1}\right),\left(\min \overline{\bar{\mu}}_{A^{-} W}\left(C_{y}\right) \mid y \in Y_{2}\right)\right) \\
& \overline{\bar{u}}_{A^{+} W}\left(C_{y}\right)=\left(\left(\max \overline{\bar{u}}_{A^{+} W}\left(C_{y}\right) \mid y \in Y_{1}\right),\left(\min \overline{\bar{u}}_{A^{-W}}\left(C_{y}\right) \mid y \in Y_{2}\right)\right) \\
& \overline{\bar{\mu}}_{A^{-} W}\left(C_{y}\right)=\left(\left(\max \overline{\bar{\mu}}_{A^{-} W}\left(C_{y}\right) \mid y \in Y_{1}\right),\left(\min \overline{\bar{\mu}}_{A^{-} W}\left(C_{y}\right) \mid y \in Y_{2}\right)\right) \\
& \overline{\bar{u}}_{A^{-} W}\left(C_{y}\right)=\left(\left(\max \overline{\bar{u}}_{A^{-} W}\left(C_{y}\right) \mid y \in Y_{1}\right),\left(\min \overline{\bar{u}}_{A^{-} W}\left(C_{y}\right) \mid y \in Y_{2}\right)\right)
\end{aligned}
$$

\section{Stage 6. Calculate the distance measures}

The distance measures given in [18] are used to measure the separation between alternatives. Once the distance measure is chosen, the separation measures are calculated as $d_{I F S}\left(M_{i}, A^{+}\right), d_{I F S}\left(M_{i}, A^{-}\right)$for each alternative on the basis of intuitionistic fuzzy positive and negative-ideal solutions. Here, the intuitionistic Euclidean distance (2000) is used to rank all alternatives.

$$
d_{I F S}\left(M_{i}, A^{+}\right)=\sqrt{\sum_{y=1}^{k}\left[\left(\mu_{M_{i} W}\left(C_{y}\right)-\mu_{A^{+} W}\left(C_{y}\right)\right)^{2}+\left(u_{M_{i} W}\left(C_{y}\right)-u_{A^{+} W}\left(C_{y}\right)\right)^{2}+\left(\pi_{M_{i} W}\left(C_{y}\right)-\pi_{A^{+} W}\left(C_{y}\right)\right)^{2}\right]}
$$




$$
d_{I F S}\left(M_{i}, A^{-}\right)=\sqrt{\sum_{y=1}^{k}\left[\left(\mu_{M_{i} W}\left(C_{y}\right)-\mu_{A^{-W}}\left(C_{y}\right)\right)^{2}+\left(u_{M_{i} W}\left(C_{y}\right)-u_{A^{-W}}\left(C_{y}\right)\right)^{2}+\left(\pi_{M_{i} W}\left(C_{y}\right)-\pi_{A^{-} W}\left(C_{y}\right)\right)^{2}\right]}
$$

\section{Stage 7. Calculate the relative closeness coefficient of each alternative}

The relative closeness coefficient of an alternative $M_{i}$ with respect to the intuitionistic fuzzy positive ideal solution $A^{+}$ is defined as follows:

$$
C C_{i^{+}}=\frac{d_{I F S}\left(M_{i}, A^{-}\right)}{d_{I F S}\left(M_{i}, A^{+}\right)+d_{I F S}\left(M_{i}, A^{-}\right)}, 0 \leq C C_{i^{+}} \leq 1, i=1,2,3, \cdots, a
$$

A larger value of $\mathrm{CC}_{i^{+}}$indicates that an alternative is closer to IFPIS and farther away from IFNIS simultaneously. Therefore, the ranking order of all the alternatives can be determined according to the descending order of $C C$ values. The most preferred alternative is the one with the highest $C C$ value.

\section{Stage 8. Rank the alternatives}

After evaluating the relative closeness coefficient of each alternative, alternatives are ranked according to descending order of $\mathrm{CC}_{i^{+}}$values.

\section{Numerical Illustration}

IT organizations aim to select the most appropriate patch management tool for analyzing patches that needs to be rolled out. After pre-evaluation, five patch management software are shortlisted as alternatives. To rank the tools, a team of five decision-makers or experts is created. The three most important criteria derived from rigorous literature review are:

$P_{1}$ : Vulnerability prioritization (determining which updates will be rolled out to which devices)

$P_{2}$ : Vulnerability analysis (identifying relevant vulnerabilities and updates)

$P_{3}$ : Vendor participation (covering a wide variety of vendor software)

The selection of the patch management tool follows the given steps:

\section{Step 1 Determine the weights of the decision makers}

Firstly, the degree of importance for each decision maker is calculated using Equation (5). Table 1 presents the linguistic terms used for determining the significance of each decision maker. Table 2 and Table 3 shows the linguistic terms used by each decision maker for weighing the criteria and alternatives respectively.

$$
\begin{aligned}
& \lambda_{D M_{1}}=\frac{0.9}{0.9+\left(0.75+0.05 \times\left(\frac{0.75}{0.75+0.2}\right)\right)+0.9+\left(0.5+0.05 \times\left(\frac{0.5}{0.5+0.45}\right)\right)+\left(0.75+0.05 \times\left(\frac{0.75}{0.75+0.2}\right)\right)}=0.23 \\
& \lambda_{D M_{2}}=\frac{\left(0.75+0.05 \times\left(\frac{0.75}{0.75+0.2}\right)\right)}{0.9+\left(0.75+0.05 \times\left(\frac{0.75}{0.75+0.2}\right)\right)+0.9+\left(0.5+0.05 \times\left(\frac{0.5}{0.5+0.45}\right)\right)+\left(0.75+0.05 \times\left(\frac{0.75}{0.75+0.2}\right)\right)}=0.202 \\
& \lambda_{D M_{3}}=\frac{0.9}{0.9+\left(0.75+0.05 \times\left(\frac{0.75}{0.75+0.2}\right)\right)+0.9+\left(0.5+0.05 \times\left(\frac{0.5}{0.5+0.45}\right)\right)+\left(0.75+0.05 \times\left(\frac{0.75}{0.75+0.2}\right)\right)}=0.23
\end{aligned}
$$




$$
\lambda_{D M_{4}}=\frac{\left(0.5+0.05 \times\left(\frac{0.5}{0.5+0.45}\right)\right)}{0.9+\left(0.75+0.05 \times\left(\frac{0.75}{0.75+0.2}\right)\right)+0.9+\left(0.5+0.05 \times\left(\frac{0.5}{0.5+0.45}\right)\right)+\left(0.75+0.05 \times\left(\frac{0.75}{0.75+0.2}\right)\right)}=0.135
$$

Table 1. Importance of decision makers and their weights

\begin{tabular}{|c|c|c|c|c|c|}
\hline & 1 & 2 & 3 & 4 & 5 \\
\hline Linguistic terms & Very significant & Significant & Very significant & Medium & Significant \\
\hline & 0.23 & 0.2 & 0.23 & 0.13 & 0.2 \\
\hline
\end{tabular}

Table 2. Linguistic terms for decision makers and criteria

\begin{tabular}{|c|c|} 
Table 2. Linguistic terms for decision makers and criteria \\
\begin{tabular}{|c|c|}
\hline Linguistic terms & IFNs \\
\hline Very significant & $(0.9,0.1)$ \\
\hline Significant & $(0.75,0.2)$ \\
\hline Medium & $(0.5,0.45)$ \\
\hline Insignificant & $(0.35,0.6)$ \\
\hline Very insignificant & $(0.1,0.9)$ \\
\hline
\end{tabular}
\end{tabular}

Table 3. Linguistic terms for alternatives

\begin{tabular}{|c|c|}
\hline Linguistic terms & IFNs \\
\hline Extremely good & $(1,0)$ \\
\hline Very very good & $(0.9,0.1)$ \\
\hline Very good & $(0.8,0.1)$ \\
\hline Good & $(0.7,0.2)$ \\
\hline Medium good & $(0.6,0.3)$ \\
\hline Fair & $(0.5,0.4)$ \\
\hline Medium bad & $(0.4,0.5)$ \\
\hline Bad & $(0.25,0.6)$ \\
\hline Very bad & $(0.1,0.75)$ \\
\hline Very very bad & $(0.1,0.9)$ \\
\hline
\end{tabular}

\section{Step 2 Construct the aggregated intuitionistic fuzzy decision matrix based on the opinions of decision makers}

The ratings provided by the decision makers (as shown in Table 4) are combined to create the intuitionistic fuzzy decision matrix through Equation (7) as shown in Table 5.

\begin{tabular}{|c|c|c|c|c|c|c|}
\hline \multirow{2}{*}{ Criteria } & \multirow{2}{*}{ Patch management solutions } & \multicolumn{5}{|c|}{ Decision makers } \\
\hline & & $D M_{1}$ & $D M_{2}$ & $\mathrm{DM}_{3}$ & $\mathrm{DM}_{4}$ & $D M_{5}$ \\
\hline \multirow{5}{*}{$P_{1}$} & SolarWinds & $\mathrm{G}$ & VG & $\mathrm{G}$ & EG & VVG \\
\hline & LANDesk & MG & $\mathrm{G}$ & $\mathrm{F}$ & $\mathrm{VG}$ & $\mathrm{G}$ \\
\hline & Shavlik & VVG & VG & $\mathrm{VG}$ & VVG & $\mathrm{G}$ \\
\hline & ManageEngine Desktop Central & MG & $\mathrm{G}$ & $\mathrm{G}$ & MG & $\mathrm{F}$ \\
\hline & GFI LanGuard & $\mathrm{F}$ & MG & MG & $\mathrm{F}$ & MB \\
\hline \multirow{5}{*}{$P_{2}$} & SolarWinds & MG & $\mathrm{G}$ & MG & $\mathrm{G}$ & VG \\
\hline & LANDesk & $\mathrm{F}$ & MG & $\mathrm{G}$ & $\mathrm{F}$ & $\mathrm{G}$ \\
\hline & Shavlik & VG & $\mathrm{G}$ & VG & G & VG \\
\hline & ManageEngine Desktop Central & $\mathrm{F}$ & $\mathrm{F}$ & MG & G & MG \\
\hline & GFI LanGuard & MB & $\mathrm{F}$ & $\mathrm{F}$ & VB & $\mathrm{B}$ \\
\hline \multirow{5}{*}{$P_{3}$} & SolarWinds & VG & $\mathrm{G}$ & VG & VVG & VG \\
\hline & LANDesk & $\mathrm{G}$ & MG & MG & EG & MG \\
\hline & Shavlik & VG & VG & $\mathrm{G}$ & EG & VVG \\
\hline & ManageEngine Desktop Central & VG & $\mathrm{G}$ & $\mathrm{G}$ & $\mathrm{G}$ & VVG \\
\hline & GFI LanGuard & $\mathrm{G}$ & $\mathrm{G}$ & $\mathrm{MG}$ & $\mathrm{G}$ & $\mathrm{F}$ \\
\hline
\end{tabular}

Table 4. Ratings of the alternatives 
Table 5. Weights assigned to each alternative

\begin{tabular}{|c|c|c|c|}
\hline & $P_{1}$ & $P_{2}$ & $P_{3}$ \\
\hline SolarWinds & $(1,0,0)$ & $(0.68,0.213,0.106)$ & $(0.799,0.118,0.084)$ \\
\hline LANDesk & $(0.684,0.239,0.077)$ & $(0.613,0.283,0.104)$ & $(1,0,0)$ \\
\hline Shavlik & $(0.828,0.118,0.054)$ & $(0.768,0.129,0.104)$ & $(1,0,0)$ \\
\hline ManageEngine Desktop Central & $(0.627,0.27,0.103)$ & $(0.572,0.326,0.102)$ & $(0.778,0.151,0.071)$ \\
\hline GFI LanGuard & $(0.563,0.383,0.054)$ & $(0.385,0.5,0.115)$ & $(0.641,0.256,0.103)$ \\
\hline
\end{tabular}

\section{Step 3 Weights of criteria using entropy}

Weights computed using Equations (8)-(10) are shown in Table 6.

\begin{tabular}{|} 
Table 6. Weights of the criteria \\
\begin{tabular}{|c|c|c|c|}
\hline Criteria & $P_{1}$ & $P_{2}$ & $P_{3}$ \\
\hline Entropy $\left(E_{L T}^{I F S}\left(C_{j}\right)\right)$ & 0.7957 & 0.8793 & 0.7285 \\
\hline Distance measure $\left(d_{j}\right)$ & 0.2043 & 0.1207 & 0.2715 \\
\hline Weights $\left(w_{j}\right)$ & 0.3425 & 0.2024 & 0.4551 \\
\hline
\end{tabular}
\end{tabular}

\section{Step 4 Construct the aggregated weighted intuitionistic fuzzy decision matrix}

Equation (11) is used to aggregate the weighted intuitionistic fuzzy decision matrix, as shown in Table 7.

Table 7. Aggregated weighted IFS-entropy decision matrix

\begin{tabular}{|c|c|c|c|c|c|c|c|c|c|c|}
\hline \multirow{2}{*}{\multicolumn{2}{|c|}{ Stage 4}} & \multicolumn{3}{|c|}{$P_{1}$} & \multicolumn{3}{|c|}{$P_{2}$} & \multicolumn{3}{|c|}{$P_{3}$} \\
\hline & & $\mu$ & $u$ & $\pi$ & $\mu$ & $u$ & $\pi$ & $\mu$ & $u$ & $\pi$ \\
\hline \multirow{5}{*}{$\begin{array}{c}\text { Aggregated } \\
\text { weighted IFS- } \\
\text { Entropy } \\
\text { Decision } \\
\text { Matrix }\end{array}$} & SolarWinds & 0.2054 & 0.7669 & 0.0277 & 0.0719 & 0.9014 & 0.0267 & 0.5188 & 0.3906 & 0.0906 \\
\hline & LANDesk & 0.1145 & 0.8513 & 0.0342 & 0.0632 & 0.9117 & 0.0251 & 0.6993 & 0.2763 & 0.0244 \\
\hline & Shavlik & 0.1525 & 0.814 & 0.0335 & 0.0858 & 0.8846 & 0.0296 & 0.6993 & 0.2763 & 0.0244 \\
\hline & $\begin{array}{l}\text { Manage Engine } \\
\text { Desktop Central }\end{array}$ & 0.1053 & 0.8586 & 0.0361 & 0.0591 & 0.9213 & 0.0196 & 0.5093 & 0.4218 & 0.0689 \\
\hline & GFI LanGuard & 0.0881 & 0.8886 & 0.0233 & 0.0381 & 0.945 & 0.0169 & 0.4007 & 0.5096 & 0.0897 \\
\hline
\end{tabular}

\section{Step 5 Obtain intuitionistic fuzzy positive ideal solution and intuitionistic fuzzy negative ideal solution}

Here, criteria are classified into benefit criteria and cost criteria; thus, we calculate the positive and negative ideal solutions using Equation (12), as shown in Table 8.

Table 8. IFPIS \& IFNIS

\begin{tabular}{|c|c|c|c|c|c|c|c|c|c|}
\hline \multirow{2}{*}{ Stage 5 } & \multicolumn{3}{|c|}{$P_{1}$} & \multicolumn{3}{|c|}{$P_{2}$} & \multicolumn{2}{|c|}{$P_{3}$} \\
\cline { 2 - 24 } & $\mu$ & $u$ & $\pi$ & $\mu$ & $u$ & $\pi$ & $\mu .147507$ & 0.259515 \\
\hline$A^{+}$ & 0.201139 & 0.147262 & 0.147507 & 0.259515 & 0.393396 & 0.201139 & 0.147262 & 0.1475 \\
\hline$A^{-}$ & 0.059838 & 0.151868 & 0.163756 & 0.022305 & 0 & 0.059838 & 0.151868 & 0.163756 & 0.022305 \\
\hline
\end{tabular}

\section{Step 6 Calculate the separation measures and relative closeness coefficient to the intuitionistic ideal solution}

Finally, the intuitionistic Euclidean distance is used to calculate the separation measures and closeness coefficients for each alternative through Equations (13) and (14), and the results are shown in Table 9.

\begin{tabular}{|c|c|c|c|c|c|c|}
\hline \multirow{2}{*}{$\begin{array}{c}\text { Separation } \\
\text { Measures \& } \\
\text { Closeness } \\
\text { Coefficient }\end{array}$} & $d_{I F S}\left(A_{i}, A^{+}\right)$ & 0.4485 & 0.2446 & 0.3529 & 0.4485 & 0.2446 \\
\hline & $d_{I F S}\left(A_{i}, A^{-}\right)$ & 0.3837 & 0.3897 & 0.5039 & 0.3837 & 0.3897 \\
\hline
\end{tabular}




\section{Step 7 Alternatives are ranked}

We determine the relative closeness coefficients and then rank the five alternatives in descending order of $C C_{i^{+}}$values. The alternatives were ranked as $A_{3}>A_{2}=A_{5}>A_{4}=A_{1}$ or Shavlik $>$ LANDesk $=$ GFI LanGuard $>$ ManageEngine Desktop Central $=$ SolarWinds. We can conclude that Shavlik is the most suitable patch management tool among the alternatives.

\section{Conclusions}

Many applications of intuitionistic fuzzy sets have been proposed in recent years as a multi-criteria decision-making approach in the broad areas of engineering and management. In this paper, we have evaluated and ranked the most appropriate patch management software with the help of the proposed hybrid intuitionistic entropy-based decision making model and TOPSIS. In information theory, entropy has been used as a measurement in relation to the average information available for the source. Intuitionistic fuzzy set theory first values the decision makers and evaluates the importance of each option on the basis of pre-determined criteria, and then it uses Shannon's entropy to obtain the optimal criteria weights. We have aggregated the weighted intuitionistic fuzzy and entropy-based matrix via the IFWA operator. Finally, TOPSIS methodology is used to determine the best alternative. Here, Shavlik is observed to be the most superior patch management software among others and has the capability to fulfill the mentioned criteria.

\section{References}

1. F. M. Nicastro, "Security Patch Management," Information Systems Security, Vol. 12, No. 5, pp. 5-18, 2003

2. N. Cain, A. Baron, S. Sharma, and F. Zakrajsek, U.S. Patent No. 962,769, Washington, DC: U.S. Patent and Trademark Office, 2004

3. E. Follis, "Discover the Best Patch Management Software for your Business," Search Security, 2017

4. https://one.comodo.com/patch-management/ (last accessed on 20 August 2017)

5. B. Hale, "Why Every IT Practitioner should Care about Network Change and Configuration Management," (last accessed on 23 August 2017), 2012

6. T. Steven, C. Dale, and B. Dan, "Recommended Practice for Patch Management of Control Systems," U.S. DOI: 10.2172/944885, 2008

7. B. Louis, "Cloud Desktop/Server in De Cloud," http://hdl.handle.net/10046/1006 (last accessed on 23 August 2017), 2013

8. S. M. Welberg, "Vulnerability Management Tools for COTS Software-A Comparison," Hg. v. University of Twente, online verfügbar unter http://doc. utwente. nl/64654/1/Vulnerability_management_tools_for_COTS_soft ware_-_a_comparison_v2, 1. 2008

9. F. E. Boran, S. Genç, M. Kurt, and D. Akay, "A Multi-Criteria Intuitionistic Fuzzy Group Decision Making for Supplier Selection with TOPSIS Method," Expert Systems with Applications, Vol. 36, No. 8, pp. 11363-11368, 2009

10. K. T. Atanassov, "Intuitionistic Fuzzy Sets," Fuzzy Sets and Systems, Vol. 20, No. 1, pp. 87-96, 1986

11. M. H. Shu, C. H. Cheng, and J. R. Chang, "Using Intuitionistic Fuzzy Sets for Fault-Tree Analysis on Printed Circuit Board Assembly," Microelectronics Reliability, Vol. 46, No. 12, pp. 2139-2148, 2006

12. C. E. Shannon, "A Mathematical Theory of Communication," Bell System Technical Journal, Vol. 27, No. 3, pp. 623-656, 1948

13. A. De Luca and S. Termini, "A Definition of Non-Probabilistic Entropy in the Setting of Fuzzy Entropy," Readings in Fuzzy Sets for Intelligent Systems, Vol. 5, pp. 301-312, 1972

14. E. Szmidt and J. Kacprzyk, "Intuitionistic Fuzzy Sets in some Medical Applications," in Proceedings of International Conference on Computational Intelligence, pp. 148-151, Springer, Berlin, Heidelberg, 2001

15. I. K. Vlachos and G. D. Sergiadis, "Intuitionistic Fuzzy Information-Applications to Pattern Recognition," Pattern Recognition Letters, Vol. 28, No. 2, pp. 197-206, 2007

16. Z. Xu, "Intuitionistic Fuzzy Aggregation Operators," IEEE Transactions on Fuzzy Systems, Vol. 15, No. 6, pp. 1179-1187, 2007

17. M. Zeleny and J. L. Cochrane, "Multiple Criteria Decision Making," McGraw-Hill, New York, 1982

18. K. T. Atanassov, "Intuitionistic Fuzzy Sets," Intuitionistic Fuzzy Sets, Physica, pp. 1-137, Heidelberg, 1999 